\title{
Vasitis Nodosa and Related Lesions: A Modern Immunohistochemical Staining Profile with Special Emphasis on Novel Diagnostic Dilemmas
}

Brie E Kezlarian, $\mathrm{MD}^{1}$, Liang Cheng, $\mathrm{MD}^{2}$, Nilesh S. Gupta, $\mathrm{MD}^{1}$, Sean R Williamson, $\mathrm{MD}^{1,3}$

${ }^{1}$ Department of Pathology and Laboratory Medicine and Henry Ford Cancer Institute, Henry Ford Health System, Detroit, MI, USA; ${ }^{2}$ Departments of Pathology and Laboratory Medicine and Urology, Indiana University School of Medicine, Indianapolis, IN, USA;

${ }^{3}$ Department of Pathology, Wayne State University School of Medicine, Detroit, MI, USA.

Running head: Vasitis nodosa immunohistochemistry

Address correspondence and reprint requests to:

Sean R. Williamson, MD

Henry Ford Hospital, Department of Pathology - K6

2799 West Grand Blvd

Detroit, MI 48202

Email: swilli25@hfhs.org

Phone: $313-916-3986$

Fax: 313-916-2385

Conflicts of Interest and Source of Funding: None declared

This is the author's manuscript of the article published in final edited form as:

Kezlarian, B. E., Cheng, L., Gupta, N. S., \& Williamson, S. R. (2017). Vasitis Nodosa and Related Lesions: A Modern Immunohistochemical Staining Profile with Special Emphasis on Novel Diagnostic Dilemmas. Human Pathology. https://doi.org/10.1016/j.humpath.2017.12.001 


\section{ABSTRACT}

Vasitis nodosa is a benign proliferation of vas deferens epithelium, thought to be a response to trauma or obstruction, usually vasectomy. Although histologic features mimic malignancy, diagnosis is usually straightforward due to the clinical context. We analyzed 21 specimens with vasitis or epididymitis nodosa with antibodies to PAX8, CD10, p63, alpha-methyl-acyl-coA-racemase (AMACR), GATA3, prostein, NKX3.1, and prostatespecific antigen (PSA). Two diagnostically problematic cases included 1) florid bladder muscle involvement after prostatectomy and 2) involvement of the ampulla and ejaculatory duct in a radical prostatectomy specimen. Vasitis nodosa was excluded in 3 additional histologic mimics ( 2 post-treatment prostate cancers and 1 bladder cancer). PAX8 yielded consistent positive $(100 \%)$ nuclear staining in the proliferative glands of vasitis nodosa, often stronger and more uniform than native vas deferens. CD10 labeling was common but also labeled secretions and other structures. Labeling for p63 was often basally located in glands with a multilayered appearance, but often markedly attenuated or lacking in the proliferative glands compared to native epithelium. AMACR positivity was variable but often present (19/21). PSA, prostein, and NKX3.1 were consistently negative. Rare problematic cases of vasitis nodosa include "invasion" of the ejaculatory duct at the prostate and involvement of bladder muscle after prostatectomy. The proliferative vasitis nodosa glands often have a prostate cancer-like staining pattern with variable AMACR positivity and negative or patchy p63. However, reliable positivity for PAX8, patchy GATA3, and negative staining for PSA, NKX3.1, and prostein aid in distinguishing from prostate cancer and tubular variants of bladder cancer. 
Key words: Vasitis nodosa, immunohistochemistry, prostate cancer, GATA3, PAX8, NKX3.1

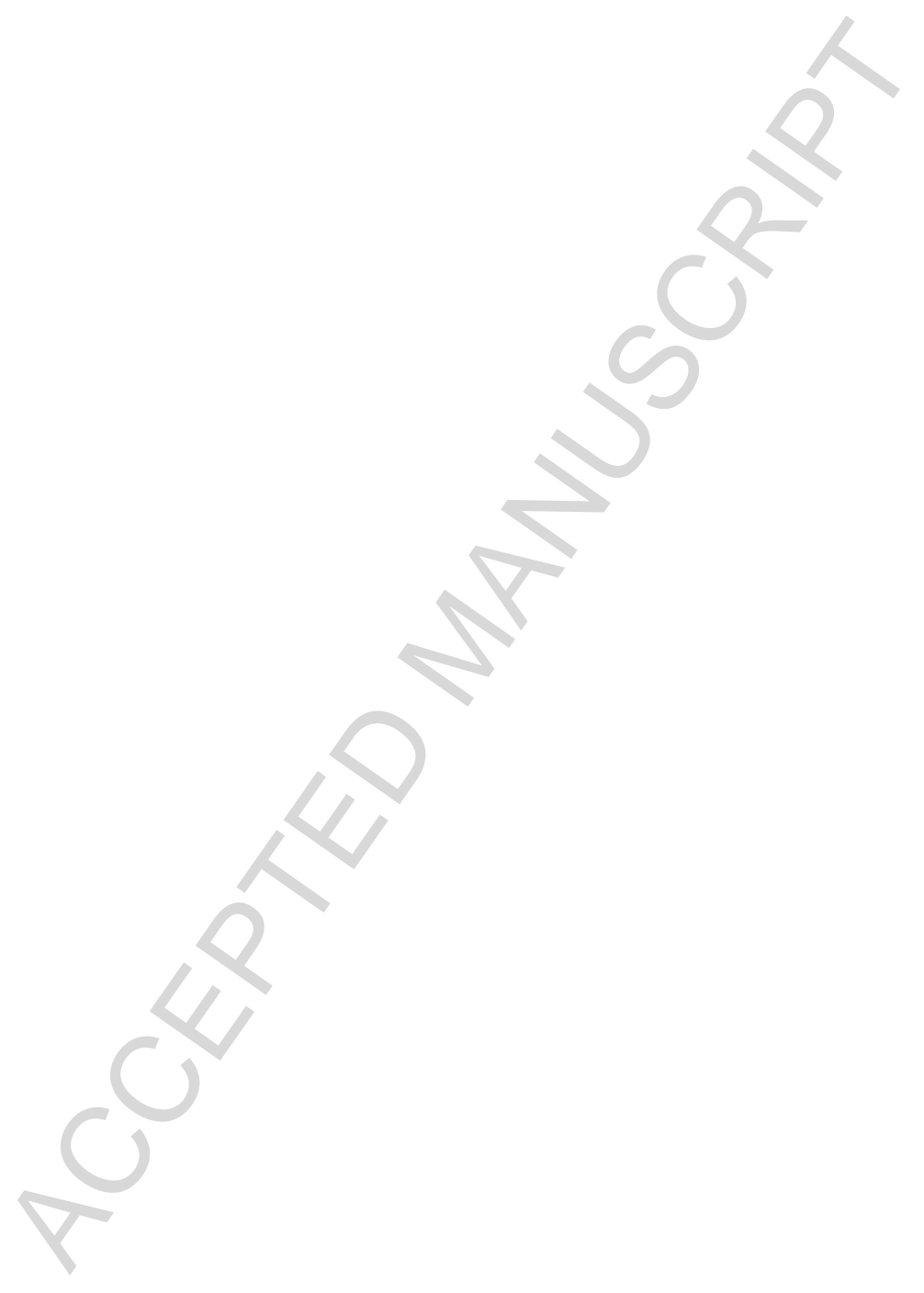




\section{Introduction}

Vasitis nodosa is a benign proliferation of vas deferens epithelium, thought to occur as a response to mechanical obstruction or traumatic injury. It is hypothesized that mechanical obstruction leads to increased intraluminal pressure and resulting spermatic fluid leakage. This extravasation of fluid is thought to result in subsequent inflammation and glandular proliferation. Vasitis nodosa typically occurs in the setting of prior vasectomy, but has been reported in other clinical scenarios, such as trauma, primary infertility, cystitis, or surgery. Clinical findings can include palpable nodules, scrotal pain, or swelling [1-4].

In the usual clinical context (characteristically post-vasectomy), vasitis nodosa is a relatively straightforward diagnosis, despite that it is known to have worrisome histologic characteristics, such as prominent nucleoli, pseudoinvasive growth pattern, and vascular and perineural invasion [5-8]. However, we have encountered rare clinical scenarios in which vasitis nodosa presented a diagnostic challenge, including occurrence at the ampulla of the vas deferens (the tortuous and dilated segment at the junction with the prostate and ejaculatory duct) in a prostate cancer specimen and in the wall of a cystectomy specimen after radical prostatectomy. Conversely, we have encountered occasional cancers that raised morphologic consideration of vasitis nodosa in these sites. Although a few immunohistochemical markers have been previously studied vasitis nodosa, we sought to characterize the immunohistochemical staining pattern of vasitis nodosa with a modern antibody panel to aid in such scenarios. 


\section{Materials and Methods}

After approval by the Institutional Review Board of the Henry Ford Health System, we queried the databases at two academic health systems (Indiana University Health and Henry Ford Health System) for cases of vasitis nodosa, epididymitis nodosa, and sperm granuloma, yielding 29 specimens. These cases were reviewed, of which 21 from 18 patients were ultimately confirmed to have vasitis or epididymitis nodosa and were included in the final cohort.

The specimens were from primarily vasectomy, vasovasostomy, or excisional biopsy samples, whereas 2 of the problematic diagnoses were found in resection specimens ( 1 radical prostatectomy and 1 cystectomy). Three cancer specimens ( 2 prostate cancers and 1 bladder cancer) for which morphology raised consideration of vasitis nodosa were also studied. Each specimen was fixed in $10 \%$ formalin solution and embedded in paraffin wax. Sections of 4-micron thickness were cut, stained with hematoxylin and eosin, and microscopically examined.

Each specimen was scored for presence or absence pseudoinvasive growth pattern, perineural invasion, vascular invasion, and prominent nucleoli. Clinical parameters, including age, reason for surgery, history of obstructive event, and history of previous chemotherapy or radiation were gathered. Using 4 um thick tissue sections, immunohistochemistry was performed using DAKO Link Autostainer automated system (DAKO Corp). Immunohistochemical stains were performed using anti-GATA3 monoclonal mouse antibody (1:200; Biocare, Concord, CA), anti-PAX8 monoclonal mouse antibody (1:50; Biocare, Concord, CA), anti-CD10 monoclonal mouse antibody (1:75; DAKO, Carpinteria, CA), anti-prostein (p501s) monoclonal mouse antibody 
(1:1000; DAKO, Carpinteria, CA), anti-prostate-specific antigen monoclonal rabbit antibody (1:6000; DAKO, Carpinteria, CA), anti-NKX3.1 polyclonal rabbit antibody (1:50, Biocare, Concord, CA); and a PIN cocktail consisting of anti-p63 and alphamethylacyl-CoA racemase (AMACR), monoclonal mouse antibodies (Biocare, Concord, CA). Hematoxylin was used as a counterstain. Strength and pattern of staining, in addition to percent of lesional tissue staining positive were recorded.

\section{Results}

Proliferative glands with prominent nucleoli were commonly present, in 19 of 21 cases, with infrequent perineural localization (3/21 cases). In the vasitis nodosa tissues (Figures 1-2), PAX8 yielded consistent positive (100\%) nuclear staining in the lesional glands, often stronger and more uniform than native vas deferens, and GATA3 was often positive (10/18). CD10 staining, was consistently positive (21/21 cases), but in addition to the proliferative glands, this staining also labeled luminal and extra-cellular secretions, and the basement membranes and apical surfaces of native and proliferative glands. Labeling for p63 was often basally located, resembling the basal cell pattern expected of prostate glandular tissue, but often markedly attenuated or lacking in the proliferative glands compared to the native epithelium. Labeling for GATA3 largely corresponded to the same areas as p63 positivity, preferentially glands with a more multilayered appearance. Overall, 12/21 cases demonstrated p63 positive basally-located cells, although this was variable within a given specimen, ranging from $10-80 \%$ of glands positive (median $28 \%$ ). Specimens without any p63-positive cells primarily corresponded to small foci of vasitis nodosa with only a few monolayered-appearing glands. AMACR positivity was variable 
but often present (18/21), ranging from weak to strong staining. No example of vasitis nodosa included in this study was positive for PSA, prostein, or NKX3.1 (0/20). Two diagnostic challenging scenarios included: 1) a radical prostatectomy specimen from a 74year-old man, performed for Gleason 3+4=7 prostate cancer (Figure 3A-D), and 2) a cystectomy specimen from a 70-year-old man, performed for severe radiation cystitis after prostatectomy (Figure 3E-F). The cystectomy specimen demonstrated bladder muscle and florid soft tissue involvement by proliferative glands, mimicking recurrent prostate cancer invading the bladder wall. The radical prostatectomy specimen showed involvement of the ampulla and ejaculatory duct, mimicking seminal vesicle invasion.

\section{Vasitis nodosa mimics}

Three cancers for which vasitis nodosa was considered in the differential diagnosis were also studied, including 2 post-treatment prostate cancers (1 radiation and 1 androgen deprivation) and 1 post-neoadjuvant chemotherapy treated bladder cancer with a small amount of residual tumor showing gland-like tubular architecture. The possibility of vasitis nodosa was considered in both prostate cancer cases due to juxtaposition of tumor to the ejaculatory duct or seminal vesicle, 1 appearing discontinuous with the remainder of the tumor volume, likely representing treatment response of the intervening areas. These were positive for PSA (2/2), AMACR (2/2), and prostein (1/1), negative for PAX8 (0/2), p63, $(0 / 2)$ and high molecular weight keratin $(0 / 1)$.

The bladder cancer contained very little residual tumor, which exclusively had a small tubular pattern (Figure 4). Interestingly, most of the tumor in the prior transurethral resection was usual urothelial carcinoma; however, this tubular morphology was focally 
present pre-treatment. These tumor gland-like structures were negative for PAX8, PSA, and prostein, and positive for p63 and GATA3 in a patchy but substantially non-basal distribution. CD10 demonstrated patchy labeling but was negative in some of the most cytologically bland tubular areas, arguing against vasitis nodosa.

\section{Discussion}

Vasitis nodosa is a benign proliferation of the vas deferens epithelium, typically occurring post-vasectomy, or after other injury or transection. Histologic features are known to include prominent nucleoli, pseudoinvasive growth pattern, and perineural invasion, resembling prostate cancer. However, the clinical context is usually straightforward, since patients often have a known history of vasectomy and no known prostate cancer, and since specimens usually represent tissue quite a distance from the prostate. Nevertheless, we have identified in this study 2 cases in which vasitis nodosa was found in a worrisome clinical scenario, mimicking malignancy, and 3 cases of prostate or bladder cancer raising morphologic consideration of vasitis nodosa, prompting us to establish an immunohistochemical profile of vasitis nodosa using a modern antibody panel.

The main immunohistochemical marker in contemporary practice that has been previously studied in vasitis nodosa is CD10, which has been found to be consistently positive in native vas deferens [9] as well as vasitis nodosa [10]; however, this is probably of limited diagnostic utility, as it also labels basement membranes and apical surface of the native and proliferative epithelia and luminal and extra-cellular extravasated fluid as well. Interestingly, we found native vas deferens to demonstrate a distinct basal-like layer of p63 positive cells, whereas the proliferative glands had variable positivity, including sometimes 
a substantial number of small glands without p63-positive cells. Even within the same surgical specimen, smaller glands with a monolayered appearance sometimes lacked p63staining cells, whereas larger glands with multilayered epithelium demonstrated p63 staining like the native vas deferens.

PAX8 is a member of the paired box family of genes, and it is involved in the development of tissues of the Wollfian (mesonephric) duct, kidney, Mullerian organs, and the thyroid [11]. As the vas deferens is a Wolffian structure [12], the positivity for PAX8 confirmed by this study may be of value in distinguishing vasitis nodosa from other epithelial proliferations, such as prostate cancer or a bladder cancer variant. PAX8 has been demonstrated to be consistently negative in adenocarcinoma of the prostate, and usually negative in urothelial carcinomas [13-15]. Other studies have noted PAX8 positivity in the normal vas deferens $[11,12]$, and PAX2 labeling in related structures [16]. However, it is interesting that in the current study of vasitis nodosa, the staining characteristics of the proliferative vasitis nodosa glands differ somewhat from that of the normal vas deferens, particularly in that PAX8 labeling was sometimes stronger and more uniform and that GATA3 and p63 labeling can be decreased or absent. GATA3 is a transcription factor with critical roles in a variety of tissues [17], including development of Wolffian duct structures [18]. It has been recently found to be a helpful marker, although multispecific, for a variety of tumors, especially urothelial carcinoma and breast carcinoma, with consistent negative staining in prostate cancer [19-21].

This study also reveals a previously undocumented potential diagnostic pitfall in this differential diagnosis. The combination of AMACR positivity and absence of basal cells with p63 staining is frequently employed in the diagnosis of prostate cancer. A 
subset of the benign glands of vasitis nodosa can demonstrate a prostate cancer-like staining pattern, with moderate to strong AMACR and lack of p63 positive cell layer. Indeed, 2 cases included in the studied occurred in scenarios where prostate cancer was a major consideration, 1 in a radical prostatectomy for cancer, in which the proliferation occurred ampulla of the vas deferens (the tortuous and dilated segment at the junction with the prostate and ejaculatory duct), mimicking seminal vesicle invasion (pT3b disease) and 1 in a cystectomy specimen for radiation cystitis after prostatectomy, in which the proliferation mimicked recurrent cancer invading bladder wall. To our knowledge, these scenarios have not been previously reported, but they may represent uncommon contexts in which a proliferation of vas deferens occurs due to transection (in the case of prior prostatectomy) or obstruction (for unknown reasons in the radical prostatectomy case). Occurrence at the prostate is likely quite rare, as we identified only 1 case from hundreds of radical prostatectomy specimens evaluated in our institution annually, although it is also possible that subtle forms are underrecognized.

Other considerations that may be entertained for vasitis nodosa involving the bladder wall include deceptive urothelial carcinoma variants [22, 23], such as urothelial carcinoma with small tubules $[24,25]$, or microcytic urothelial carcinoma $[26,27]$. This difficulty could be further compounded by the fact that vasitis nodosa can be GATA3 and p63 positive, although as we have shown here, often in a patchy distribution. Awareness of these staining patterns and the consistent PAX8 positive, PSA, prostein, and NKX3.1negative phenotype of vasitis nodosa may aid the practicing pathologist in avoiding the potential pitfall of misdiagnosis of vasitis nodosa as malignancy. Conversely, we also report here 3 examples of malignancy that raised differential diagnostic consideration of 
vasitis nodosa, all in the post-treatment setting, including 2 prostate cancers in the ejaculatory duct area and 1 bladder cancer with small tubular structures. For prostate cancer, the positivity for PSA, NKX3.1, or prostein, combined with negative PAX8 and lack of basal distribution of p63 positive cells may be helpful in such a context. Similarly, awareness of these staining patterns, may be helpful in arguing against consideration of vasitis nodosa in the context of a tubular variant of bladder cancer.

\section{Conclusions}

In addition to characterizing the immunohistochemical signature of vasitis nodosa, this study unearthed novel diagnostic pitfalls. Rare problematic lesions include "invasion" of the ejaculatory duct at the prostate and involvement of bladder muscle after prostatectomy. Proliferative vasitis nodosa glands often have a prostate cancer-like staining pattern with variable AMACR positivity and negative or patchy $\mathrm{p} 63$, in contrast to the native vas deferens. GATA3 is also often positive, generally in a similar distribution to p63. Reliable positivity for PAX8 and negative staining for PSA, NKX3.1, and prostein aid in distinguishing vasitis nodosa from prostate cancer.

Acknowledgements: Sources of funding: none. 


\section{References}

1. Easley S, MacLennan GT. Vasitis and epididymitis nodosa. J Urol 2006; 175, 1502.

2. Sakaki M, Hirokawa M, Horiguchi H, Wakatsuki S, Sano T. Vasitis nodosa: immunohistochemical findings--case report. APMIS 2000; 108, 283-6.

3. Oliva E, Young RH. Paratesticular tumor-like lesions. Semin Diagn Pathol 2000; $17,340-58$.

4. Hirschowitz L, Rode J, Guillebaud J, Bounds W, Moss E. Vasitis nodosa and associated clinical findings. J Clin Pathol 1988; 41, 419-23.

5. Kovi J, Agbata A. Letter: Benign neural invasion in vasitis nodosa. JAMA 1974; $228,1519$.

6. Balogh K, Travis WD. The frequency of perineurial ductules in vasitis nodosa. Am J Clin Pathol 1984; 82, 710-3.

7. Balogh K, Travis WD. Benign vascular invasion in vasitis nodosa. Am J Clin Pathol 1985; 83, 426-30.

8. DeSchryver-Kecskemeti K, Balogh K, Neet KE. Nerve growth factor and the concept of neural-epithelial interactions. Immunohistochemical observations in two cases of vasitis nodosa and six cases of prostatic adenocarcinoma. Arch Pathol Lab Med 1987; $111,833-5$.

9. Sasaki K, Bastacky SI, Zynger DL, Parwani AV. Use of immunohistochemical markers to confirm the presence of vas deferens in vasectomy specimens. Am J Clin Pathol $2009 ; 132,893-8$. 
10. Cerilli LA, Sotelo-Avila C, Mills SE. Glandular inclusions in inguinal hernia sacs: morphologic and immunohistochemical distinction from epididymis and vas deferens. Am J Surg Pathol 2003; 27, 469-76.

11. Tong GX, Memeo L, Colarossi C, Hamele-Bena D, Magi-Galluzzi C, Zhou M, et al. PAX8 and PAX2 immunostaining facilitates the diagnosis of primary epithelial neoplasms of the male genital tract. Am J Surg Pathol 2011; 35, 1473-83.

12. Magers MJ, Udager AM, Chinnaiyan AM, French D, Myers JL, Jentzen JM, et al. Comprehensive Immunophenotypic Characterization of Adult and Fetal Testes, the Excretory Duct System, and Testicular and Epididymal Appendages. Appl Immunohistochem Mol Morphol 2016; 24, e50-68.

13. Laury AR, Perets R, Piao H, Krane JF, Barletta JA, French C, et al. A comprehensive analysis of PAX8 expression in human epithelial tumors. Am J Surg Pathol $2011 ; 35,816-26$.

14. Tacha D, Zhou D, Cheng L. Expression of PAX8 in normal and neoplastic tissues: a comprehensive immunohistochemical study. Appl Immunohistochem Mol Morphol $2011 ; 19,293-9$.

15. Tong GX, Yu WM, Beaubier NT, Weeden EM, Hamele-Bena D, Mansukhani MM, et al. Expression of PAX8 in normal and neoplastic renal tissues: an immunohistochemical study. Mod Pathol 2009; 22, 1218-27.

16. Quick CM, Gokden N, Sangoi AR, Brooks JD, McKenney JK. The distribution of PAX-2 immunoreactivity in the prostate gland, seminal vesicle, and ejaculatory duct: comparison with prostatic adenocarcinoma and discussion of prostatic zonal embryogenesis. Hum Pathol 2010; 41, 1145-9. 
17. Chou J, Provot S, Werb Z. GATA3 in development and cancer differentiation: cells GATA have it! J Cell Physiol 2010; 222, 42-9.

18. Grote D, Boualia SK, Souabni A, Merkel C, Chi X, Costantini F, et al. Gata3 acts downstream of beta-catenin signaling to prevent ectopic metanephric kidney induction. PLoS Genet 2008; 4, e1000316.

19. Higgins JP, Kaygusuz G, Wang L, Montgomery K, Mason V, Zhu SX, et al. Placental S100 (S100P) and GATA3: markers for transitional epithelium and urothelial carcinoma discovered by complementary DNA microarray. Am J Surg Pathol 2007; 31, 673-80.

20. Miettinen M, McCue PA, Sarlomo-Rikala M, Rys J, Czapiewski P, Wazny K, et al. GATA3: a multispecific but potentially useful marker in surgical pathology: a systematic analysis of 2500 epithelial and nonepithelial tumors. Am J Surg Pathol 2014; 38, 13-22.

21. Ordonez NG. Value of GATA3 immunostaining in tumor diagnosis: a review. Adv Anat Pathol 2013; 20, 352-60.

22. Amin MB. Histological variants of urothelial carcinoma: diagnostic, therapeutic and prognostic implications. Mod Pathol 2009; 22 Suppl 2, S96-S118.

23. Williamson SR, Lopez-Beltran A, Montironi R, Cheng L. Glandular lesions of the urinary bladder:clinical significance and differential diagnosis. Histopathology 2011; 58, 811-34.

24. Young RH, Oliva E. Transitional cell carcinomas of the urinary bladder that may be underdiagnosed. A report of four invasive cases exemplifying the homology between neoplastic and non-neoplastic transitional cell lesions. Am J Surg Pathol 1996; 20, 144854. 
25. Huang Q, Chu PG, Lau SK, Weiss LM. Urothelial carcinoma of the urinary bladder with a component of acinar/tubular type differentiation simulating prostatic adenocarcinoma. Hum Pathol 2004; 35, 769-73.

26. Young RH, Zukerberg LR. Microcystic transitional cell carcinomas of the urinary bladder. A report of four cases. Am J Clin Pathol 1991; 96, 635-9.

27. Lopez Beltran A, Montironi R, Cheng L. Microcystic urothelial carcinoma: morphology, immunohistochemistry and clinical behaviour. Histopathology 2014; 64, 8729. 


\section{Figure legends:}

Figure 1: This case of vasitis nodosa (A, 200×, hematoxylin and eosin) demonstrates the native vas deferens lumen at right and proliferative glands at left. Higher magnification demonstrates variably monolayered to multilayered epithelium with clear to eosinophilic cytoplasm and prominent nucleoli (B, 400×, hematoxylin and eosin). Staining for PAX8 (C, 200×, anti-PAX8 immunohistochemistry) labels the vasitis nodosa (left) and native lumen (right). Antibody to p63 (D, nuclear staining in dual antibody, single color cocktail, 200x, anti-p63 and alpha-methylacyl-CoA racemase immunohistochemistry) reveals a p63-positive, basally-located cell layer in the native vas deferens (right) but not the vasitis nodosa (left). Staining for alpha-methyl-acyl-coA-racemase (cytoplasmic labeling) shows similar intensity in both areas. Staining for GATA3 (E, 200×, anti-GATA3 immunohistochemistry) labels the native vas deferens lumen (right) but not the proliferative glands (left). CD10 (F, 200×, anti-CD10 immunohistochemistry) staining labels the apical cytoplasm and faintly the basal layer of the native vas deferens (right) but strongly labels all vasitis nodosa glands throughout the cells (left).

Figure 2: Vasitis nodosa glands from a vasovasostomy (vasectomy reversal) (A, 200x magnification hematoxylin and eosin) demonstrate a pseudoinfiltrative growth pattern and cytologic atypia with prominent nucleoli; however, sperm are visible in lumina (B, 400x magnification hematoxylin and eosin). Dual antibody, single color antibody cocktail for p63 and alpha-methyl-acyl-coA-racemase (AMACR, C, 200×) reveals a basally-located, p63-positive cell layer in a few glands at left; however, the proliferative glands more distant from the native lumen have no such layer and exhibit strong labeling for AMACR, 
mimicking prostatic adenocarcinoma (right). Staining for PAX8 labels the majority of vasitis nodosa glands consistently (D, 200×, anti-PAX8 immunohistochemistry). The same field with anti-GATA immunohistochemical staining (E, 200×) shows focal GATA3 labeling at left, similar to p63, but absence at right. Staining for NKX3.1 (F, anti-NKX3.1 immunohistochemistry, 200x) is entirely negative.

Figure 3: In this example of vasitis nodosa mimicking seminal vesicle invasion $(\mathrm{A}, 100 \times$, hematoxylin and eosin), the vasitis nodosa glands (left) are located near the ejaculatory duct (upper right). There is amyloid deposition around the ejaculatory duct epithelium but not the vasitis nodosa. At high magnification, the vasitis nodosa glands have prominent nucleoli (B, 400×, hematoxylin and eosin), mimicking prostate cancer. Vasitis nodosa glands demonstrate uniform nuclear staining for PAX8 (C, 400×, anti-PAX8 immunohistochemistry) and a subset of glands have p63-positive cells (D, bottom, nuclear, 400x, anti-p63 and alpha-methylacyl-CoA racemase single-color immunohistochemistry), with both p63-positive and negative glands showing substantial staining for alpha-methylacyl-coA-racemase (D cytoplasmic). In another case of vasitis nodosa infiltrating the bladder wall after prostatectomy (E, 100×, hematoxylin and eosin), glands with prominent nucleoli are present between muscularis propria bundles (F, 400×, hematoxylin and eosin).

Figure 4: In this case of urothelial carcinoma with small tubules, raising consideration of vasitis nodosa, small tubular structures infiltrate the muscularis propria (A, 200×, B, 400×, hematoxylin and eosin). In contrast to the vasitis nodosa cases, staining for p63 varies from patchy (C, 200×, anti-p63 immunohistochemistry) to diffuse (D, 200×, anti-p63 
immunohistochemistry) in a non-basal distribution. There is diffuse, staining for GATA3, even in gland-like structures with a cuboidal, monolayered appearance (E, 200×, antiGATA3 immunohistochemistry). Staining for PAX8 (F, 400×, anti-PAX8 immunohistochemistry) is entirely negative. 


\section{ACCEPTED MANUSCRIPT}

Kezlarian et al - Vasitis nodosa immunohistochemistry
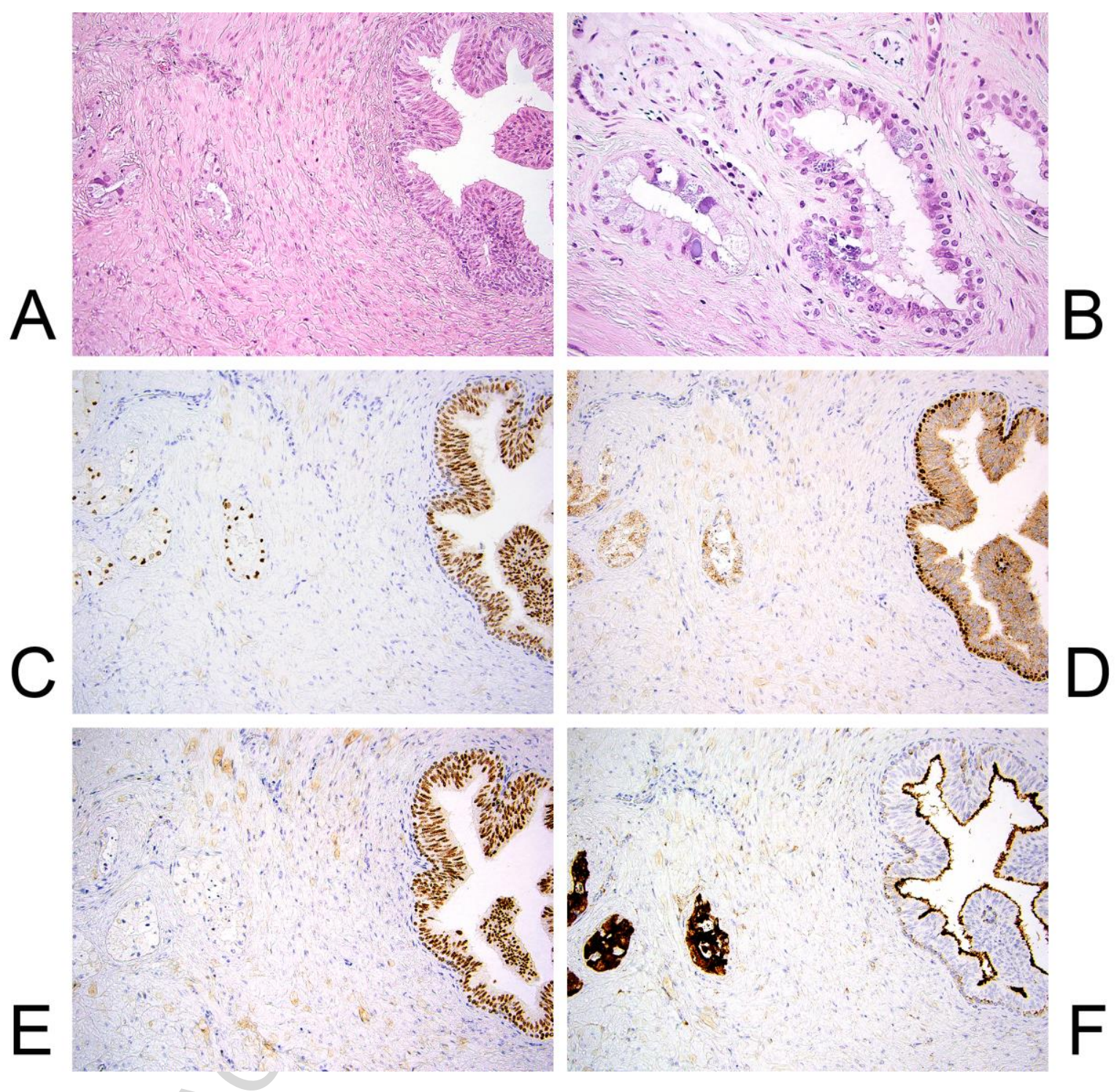

Fig. 1 


\section{ACCEPTED MANUSCRIPT}

Kezlarian et al - Vasitis nodosa immunohistochemistry
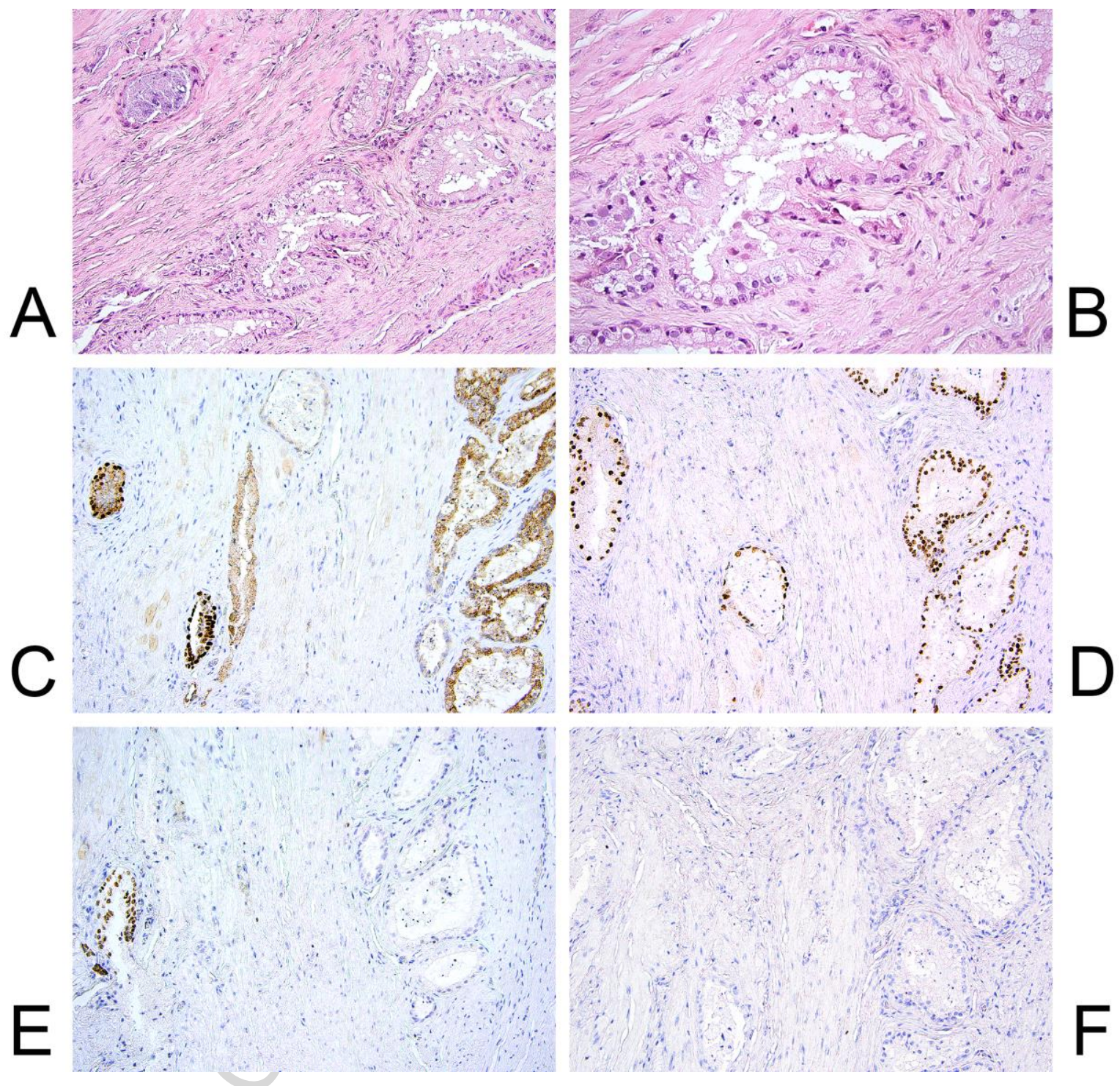

Fig. 2 


\section{ACCEPTED MANUSCRIPT}

Kezlarian et al - Vasitis nodosa immunohistochemistry
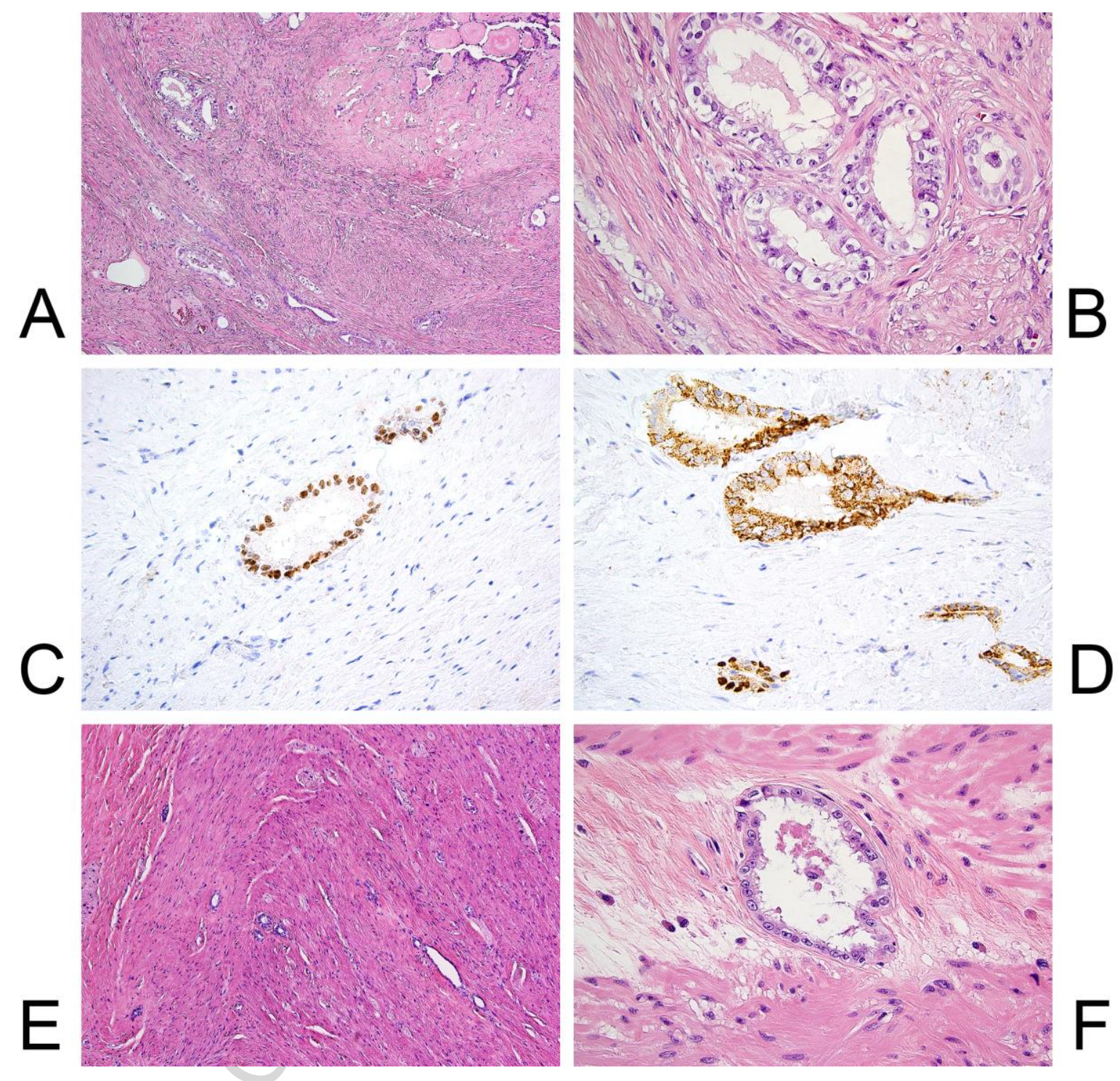

Fig. 3 


\section{ACCEPTED MANUSCRIPT}

Kezlarian et al - Vasitis nodosa immunohistochemistry
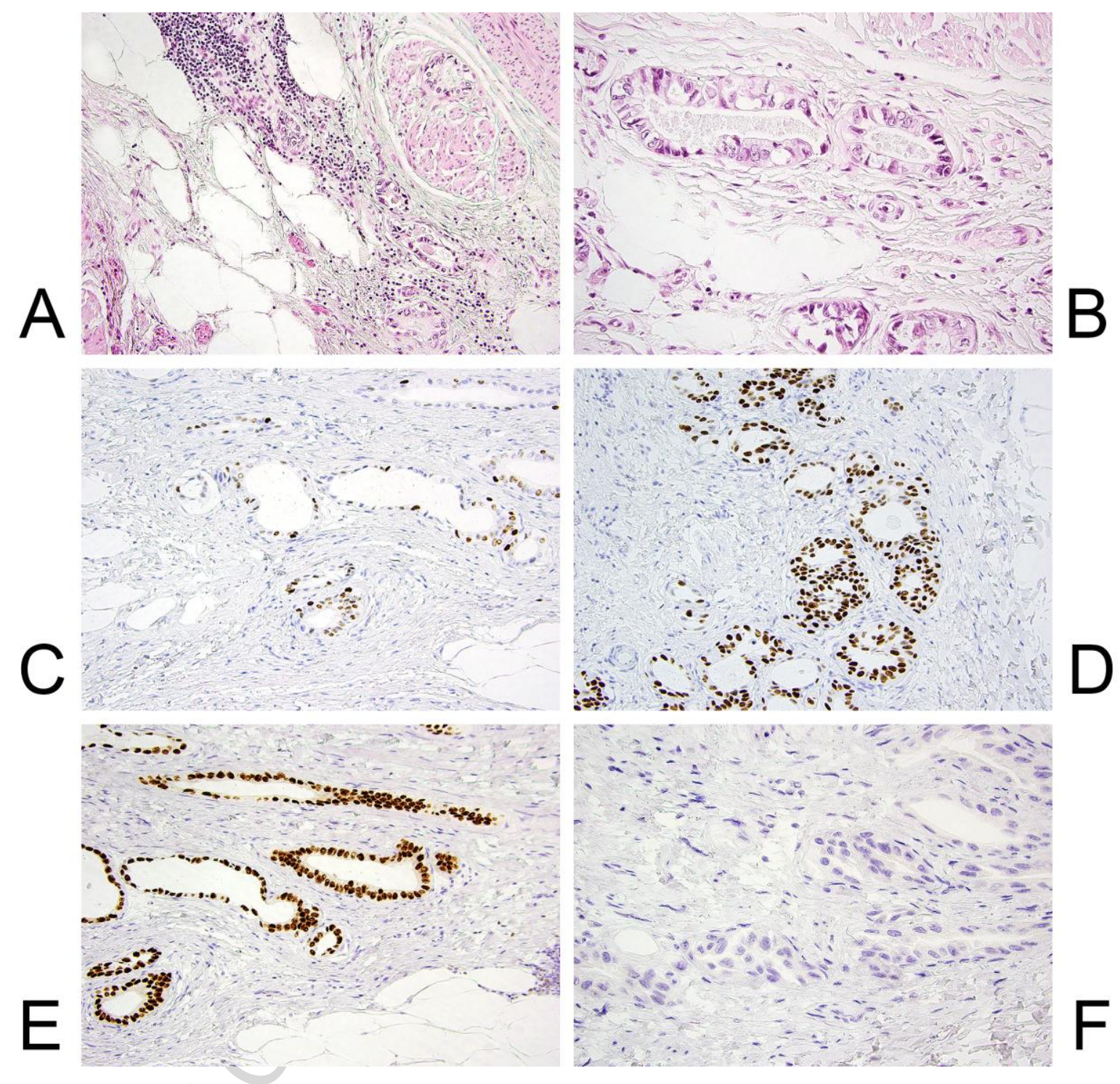

) -

D

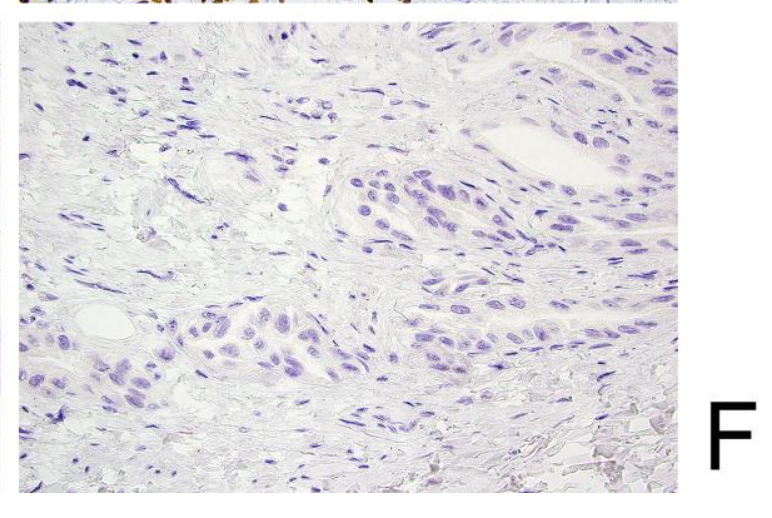

Fig. 4 
ACCEPTED MANUSCRIPT

Kezlarian et al - Vasitis nodosa immunohistochemistry

\begin{tabular}{|c|c|c|c|}
\hline & Vasitis nodosa & $\begin{array}{c}\text { Prostate cancer mimicking } \\
\text { vasitis nodosa }\end{array}$ & $\begin{array}{c}\text { Urothelial carcinoma } \\
\text { mimicking vasitis nodosa }\end{array}$ \\
\hline p63 & $12 / 21$, often basally located & $0 / 2$ & $1 / 1$, patchy \\
\hline AMACR & $\begin{array}{l}18 / 21 \text {, variable, weak to } \\
\text { strong }\end{array}$ & $2 / 2 \operatorname{ctc}$ & ND \\
\hline GATA3 & $10 / 18$, patchy or basal & ND & $1 / 1$, patchy, nonbasal \\
\hline PAX8 & $\begin{array}{c}21 / 21, \text { more uniform in } \\
\text { vasitis nodosa than normal } \\
\text { lumen }\end{array}$ & $0 / 2$ & $0 / 1$ \\
\hline CD10 & $\begin{array}{c}\text { 21/21, diffuse in glands and } \\
\text { secretions }\end{array}$ & ND & $\begin{array}{c}1 / 1, \text { patchy but some } \\
\text { cuboidal glands entirely } \\
\text { negative }\end{array}$ \\
\hline PSA & $0 / 20$ & $2 / 2$ & $0 / 1$ \\
\hline Prostein & & $1 / 1$ & $0 / 1$ \\
\hline NKX3.1 & & ND & ND \\
\hline
\end{tabular}

Table 1: Summary of immunohistochemical markers in vasitis nodosa and mimics. Abbreviations: AMACR - alpha-methylacyl-CoA racemase; PSA - prostate-specific antigen; ND - not done 


\section{Highlights}

- Vasitis nodosa is a benign proliferation with worrisome histologic features

- Immunohistochemical characteristics can mimic prostate cancer (AMACR+, p63-)

- However, glands consistently label for PAX8 and not for prostate markers

- Labeling for GATA3 and p63 is usually basal, but lacking in small glands 